\title{
A New Legendre Spectral Galerkin and Pseudo-Spectral Approximations for Fractional Initial Value Problems
}

\author{
A. H. Bhrawy ${ }^{1,2}$ and M. A. Alghamdi ${ }^{1}$ \\ ${ }^{1}$ Department of Mathematics, Faculty of Science, King Abdulaziz University, Jeddah 21589, Saudi Arabia \\ ${ }^{2}$ Department of Mathematics, Faculty of Science, Beni-Suef University, Beni-Suef 62511, Egypt \\ Correspondence should be addressed to A. H. Bhrawy; alibhrawy@yahoo.co.uk
}

Received 1 May 2013; Accepted 17 June 2013

Academic Editor: Dumitru Baleanu

Copyright (c) 2013 A. H. Bhrawy and M. A. Alghamdi. This is an open access article distributed under the Creative Commons Attribution License, which permits unrestricted use, distribution, and reproduction in any medium, provided the original work is properly cited.

\begin{abstract}
We extend the application of the Galerkin method for treating the multiterm fractional differential equations (FDEs) subject to initial conditions. A new shifted Legendre-Galerkin basis is constructed which satisfies exactly the homogeneous initial conditions by expanding the unknown variable using a new polynomial basis of functions which is built upon the shifted Legendre polynomials. A new spectral collocation approximation based on the Gauss-Lobatto quadrature nodes of shifted Legendre polynomials is investigated for solving the nonlinear multiterm FDEs. The main advantage of this approximation is that the solution is expanding by a truncated series of Legendre-Galerkin basis functions. Illustrative examples are presented to ensure the high accuracy and effectiveness of the proposed algorithms are discussed.
\end{abstract}

\section{Introduction}

Many practical problems arising in engineering, physical, biological, and biomedical sciences require solving fractional differential equations (FDEs), (see, e.g., [1-4]). For that reason, accurate and efficient numerical approaches for solving FDEs are needed. Several methods have also been proposed in the literature to solve ordinary or partial fractional differential equations (see, for instance, [5-8]). In contrast, there is a relatively small literature on spectral methods for direct solution of such fractional-order problems, (see, for instance, [9-11]).

The aim of this paper is to design some spectral techniques based on the shifted Legendre-Galerkin (SLG) method and shifted Legendre-Gauss-Lobatto collocation (SL-G-LC) method in modal basis for the solution of linear and nonlinear multi-term FDEs, respectively. Indeed, this is the first work concerning the spectral Galerkin method and pseudospectral method in modal basis for solving such problems.

In the tau, Galerkin, or pseudo-spectral approximations, the spectral solution is represented by a truncated series of smooth global trial functions, in such a representation the coefficients of the expansion are the unknown to be determined. An explicit expression for the derivatives of an infinitely differentiable function of any degree and for any fractional order in terms of the function itself is needed for tackling FDEs. In this direction, Doha et al. [9] have derived such a formula in the case of the trial functions of truncated expansion that are Chebyshev polynomials and implemented such a relation for solving two classes of FDEs. Furthermore, the fractional derivative of shifted Jacobi polynomials is derived in [12]. Ahmadian et al. [13] proposed an accurate and reliable computational scheme based Jacobi polynomials for fuzzy linear FDEs.

The pseudo-spectral methods for the numerical approximations of the solution of several types of FDEs have been proposed and developed. Maleki et al. [14] proposed an efficient and accurate pseudo-spectral method based on shifted Legendre-Gauss quadrature nodes for solving a class of FDEs with boundary conditions. The authors of [15] used the spline functions methods for tackling the linear and nonlinear FDEs. The authors of [16] proposed two types of 
spectral approximations based on shifted Legendre polynomials for solving two classes of FDEs with multipoint boundary conditions. Yüzbaşı [17] proposed the Bessel pseudospectral method to introduce an approximate solution of a class of FDEs. A collocation method based on Bernstein polynomials has recently been proposed and analyzed for solving fractional order Riccati differential equation in [18]. Moreover, the authors in [11] computed the fractional derivative of the new fractional Legendre functions (FLF), also they developed an efficient spectral tau approximations based on FLF to approximate the FDEs. Recently, Bhrawy et al. [19] investigated the fractional integrals of modified generalized Laguerre operational matrix to implement a numerical solution of the integrated form of the linear FDEs on semiinfinite interval. Meanwhile, Baleanu et al. [20] proposed and developed two efficient generalized Laguerre spectral algorithms based on the operational matrix of derivative for the solution of linear and nonlinear fractional initial value problems. We refer also to the recent papers [21-27] where several numerical methods are developed to approximate the solution of various kinds of FDEs.

The fundamental goal of this paper is to develop a direct solution technique to approximate linear FDEs subject to homogeneous initial conditions, using the shifted Legendre spectral Galerkin (SLG) approximations. We start by constructing a new appropriate shifted Legendre basis functions which satisfy the homogeneous initial equations and then are used for the approximation of the fractional differential operators. We also present an explicit expression for the derivatives of any fractional order for the shifted Legendre basis functions in terms of the shifted Legendre polynomials. Moreover, the matrices corresponding to shifted LegendreGalerkin approximation are clearly described, including the modes required to impose nonhomogeneous initial conditions.

Another goal of this paper is to treat the nonlinear FDEs subject to nonhomogeneous initial conditions by implementing a new pseudo-spectral approximation based on Legendre polynomials. This approach is characterized by the representation of the solution by a truncated series of Legendre-Galerkin basis functions. The proposed technique differs from the classical pseudo-spectral approximation in that the homogeneous initial conditions are satisfied exactly. Finally, the accuracy and effectiveness of the proposed algorithms are demonstrated by some numerical examples.

The outline of the paper is as follows. Section 2 introduces necessary definitions of fractional derivatives and shifted Legendre polynomials. In Section 3, we construct an appropriate shifted Legendre basis function for initial FDEs and prove a formula that gives the fractional derivatives of the shifted Legendre basis function in terms of the shifted Legendre polynomials. In Section 4, we present and develop the Legendre-Gauss-Lobatto collocation algorithm in modal basis for solving nonlinear FDEs. In Section 5, some numerical results are discussed. Section 6 is devoted to concluding remarks.

\section{Preliminaries and Notations}

We present recall and in this section recall some properties of the fractional calculus (see, e.g., [1-4]) and Legendre polynomials.

The Riemann-Liouville fractional integral operator is given by

$$
\begin{gathered}
J^{\mu} f(x)=\frac{1}{\Gamma(\mu)} \int_{0}^{x}(x-t)^{\mu-1} f(t) d t, \quad \mu>0, x>0, \\
J^{0} f(x)=f(x) .
\end{gathered}
$$

The Caputo fractional derivatives operator is given by

$$
\begin{aligned}
D^{\mu} f(x)= & J^{m-\mu} D^{m} f(x) \\
= & \frac{1}{\Gamma(m-\mu)} \int_{0}^{x}(x-t)^{m-\mu-1} \frac{d^{m}}{d t^{m}} f(t) d t, \\
& \quad m-1<\mu \leq m, x>0,
\end{aligned}
$$

where $D^{m}$ is $m$ th order differential operator.

The set of Legendre polynomials $\left(L_{i}(x)\right)$ forms a complete $L^{2}[-1,1]$-orthogonal system, and

$$
\left\|L_{i}(x)\right\|^{2}=h_{i}=\frac{2}{2 i+1} .
$$

Let $L_{t, i}(x)=L_{i}(2 x-1), x \in[0,1]$ be the shifted Legendre polynomial of degree $i$, then it is given by

$$
L_{t, i}(x)=\sum_{k=0}^{i}(-1)^{i+k} \frac{(i+k) ! x^{k}}{(i-k) !(k !)^{2}} .
$$

Next, let $w_{t}(x)=w(x)=1$, then we define the weighted space $L_{w_{t}}^{2}[0,1]$ in the usual way, with the following inner product and norm:

$$
(u, v)_{w_{t}}=\int_{0}^{1} u(x) v(x) w_{t}(x) d x, \quad\|u\|_{w_{t}}=(u, u)_{w_{t}}^{1 / 2} .
$$

The set of shifted Legendre polynomials forms a complete $L_{w_{t}}^{2}[0,1]$-orthogonal system. According to (3), we have

$$
\left\|L_{t, i}(x)\right\|_{w_{t}}^{2}=\frac{1}{2} h_{i}=\hbar_{i}
$$

The shifted Legendre expansion of a function $u(x) \in L_{w_{t}}^{2}[0,1]$ is

$$
u(x)=\sum_{j=0}^{\infty} a_{j} L_{t, j}(x),
$$

where $a_{j}$ are given by

$$
a_{j}=\frac{1}{\hbar_{j}} \int_{0}^{1} u(x) L_{t, j}(x) d x, \quad j=0,1,2, \ldots
$$

In the following theorem, we state the Caputo fractional derivative of order $\mu$ for the shifted Legendre polynomials, for more details, see [16]. 
Theorem 1 (see [16]). The Caputo fractional derivative of order $\mu$ of the shifted Legendre polynomials is given by

$$
D^{\mu} L_{t, i}(x)=\sum_{l=0}^{\infty} \Pi_{\mu}(i, l) L_{t, l}(x), \quad i=\lceil\mu\rceil,\lceil\mu\rceil+1, \ldots,
$$

where

$$
\Pi_{\mu}(i, l)=\sum_{k=\lceil\mu\rceil}^{i} \frac{(-1)^{i+k}(2 l+1)(i+k) !(k-l-\mu+1)_{l}}{(i-k) ! k ! \Gamma(k-\mu+1)(k-\mu+1)_{l+1}},
$$

and $\lceil\mu\rceil$ is the ceiling function.

\section{Legendre-Galerkin Method for Fractional IVPs}

In this section, we are interested in employing the SLG method for solving the FDE:

$$
D^{\nu} u(x)+\sum_{\sigma=1}^{r-1} \gamma_{\sigma} D^{\beta_{\sigma}} u(x)+\gamma_{r} u(x)=f(x), \quad x \in I=[0,1]
$$

subject to the homogeneous initial conditions

$$
u^{(q)}(0)=0, \quad q=0, \ldots, m-1,
$$

where $\gamma_{\sigma}(\sigma=1, \ldots, r)$ and $0<\beta_{1}<\beta_{2}<\cdots<\beta_{r-1}<$ $\nu, m-1<v \leq m$ are real constants, and $f(x)$ is a source function.

Let us present some basic notations which will be used in the sequel. We set

$$
\begin{gathered}
S_{N}=\operatorname{span}\left\{L_{t, 0}(x), L_{t, 1}(x), \ldots, L_{t, N}(x)\right\}, \\
V_{N}=\left\{v \in S_{N}: v^{(j)}(0)=0, j=0,1, \ldots, m-1\right\},
\end{gathered}
$$

where $v^{(j)}(x)$ is the $j$ th-order derivative of $v(x)$.

The shifted Legendre-Galerkin approximation to (11) and (12) is to find $u_{N} \in V_{N}$ such that

$$
\begin{aligned}
& \left(D^{\nu} u_{N}, v(x)\right)_{w_{t}}+\sum_{\sigma=1}^{r-1} \gamma_{\sigma}\left(D^{\beta_{\sigma}} u_{N}, v(x)\right)_{w_{t}}+\gamma_{r}\left(u_{N}, v(x)\right)_{w_{t}} \\
& \quad=(f, v(x))_{w_{t}, N}, \quad \forall v \in V_{N},
\end{aligned}
$$

where $(\cdot, \cdot)_{w_{t}}$, is defined in the space $L_{w_{t}}^{2}[0,1]$ and $(\cdot, \cdot)_{w_{t}, N}$ is the discrete inner product which will be defined later in (48).

The problem of approximating solutions of multi-term fractional differential equations by shifted Legendre-Galerkin approximation involves the projection onto the span of some appropriate sets of shifted Legendre basis function. The members of the basis may satisfy automatically the given initial conditions imposed on the multi-term FDEs (11). The following lemma provides a shifted Legendre basis function which satisfies the homogeneous initial conditions (12).
Lemma 2. Let one defines

$$
\eta_{i, m}(k)=\frac{(2 i+1+2 k)}{i !(i+1+2 k)} \prod_{n=1}^{i} \frac{(m-n+1)(2 k+n+1)}{(2 k+m+n+1)}
$$

with $\eta_{0, m}(k)=1$, then a linear combination of shifted Legendre polynomials

$$
\begin{aligned}
\phi_{k}(x)= & L_{t, k}(x) \\
& +\sum_{i=1}^{m} \eta_{i, m}(k) L_{t, k+i}(x), \quad k=0,1, \ldots, N-m
\end{aligned}
$$

satisfies the homogeneous initial conditions (12).

Proof. As a general rule, for fractional-order differential equations with $m$ initial conditions, one may choose the basis function $\phi_{k}(x)$, in the form (see, $[28,29]$ )

$$
\begin{aligned}
\phi_{k}(x)= & L_{t, k}(x) \\
& +\sum_{i=1}^{m} \eta_{i, m}(k) L_{t, k+i}(x), \quad k=0,1, \ldots, N-m .
\end{aligned}
$$

The coefficients $\left\{\eta_{i, m}(k)\right\}$ may be chosen such that $\phi_{k}(x)$ exactly satisfy the homogeneous initial conditions (12). In virtue of $L_{t, k}(0)=(-1)^{k}$ and

$$
D^{q} L_{t, k}(0)=\frac{(-1)^{k+q} \Gamma(1+k+q)}{\Gamma(1+k-q) \Gamma(1+q)}, \quad k \geq q, q=1,2, \ldots
$$

then the initial conditions (12) are reduced to the following system for $\left\{\eta_{i, m}(k)\right\}$ :

$$
\begin{array}{r}
\sum_{i=1}^{m}(-1)^{i} \eta_{i, m}(k)=-1, \\
\sum_{i=1}^{m}(-1)^{i} \eta_{i, m}(k) \frac{(k+q+1)_{i}}{(k-q+1)_{i}}=-1, \\
k=0,1, \ldots, N-m .
\end{array}
$$

The determinant of the previous system is different from zero, hence $\left\{\eta_{i, m}(k)\right\}$ can be uniquely determined to give

$$
\eta_{i, m}(k)=\frac{(2 i+1+2 k)}{i !(i+1+2 k)} \prod_{n=1}^{i} \frac{(m-n+1)(2 k+n+1)}{(2 k+m+n+1)} .
$$

Remark 3. The computation of the exact solution of the linear system (19) for the unknown coefficients $\left\{\eta_{i, m}(k)\right\}$ is extremely tedious by hand and we have resorted to the symbolic computation software Mathematica 8. 
If we substitute (15) into (16) it gives

$$
\begin{aligned}
& \phi_{k}(x)= L_{t, k}(x)+\sum_{i=1}^{m} \frac{(2 i+1+2 k)}{i !(i+1+2 k)} \\
& \times \prod_{n=1}^{i} \frac{(m-n+1)(2 k+n+1)}{(2 k+m+n+1)} L_{t, k+i}(x), \\
& k=0,1,2, \ldots, N-m .
\end{aligned}
$$

Now, it is clear that $\left\{\phi_{k}(x)\right\}$ are linearly independent. Therefore by dimension argument we get

$$
V_{N}=\operatorname{span}\left\{\phi_{k}(x): k=0,1,2, \ldots, N-m\right\} .
$$

In the following theorem, we introduce a formula expanding explicitly the fractional derivatives of the basis functions for any fractional-order in terms of shifted Legendre polynomials.

Theorem 4. The Caputo fractional derivative for the shifted Legendre basis functions is given by

$$
D^{\mu} \phi_{j}(x)=\sum_{\ell=0}^{\infty} \Theta_{m, \mu}(j, \ell) L_{t, \ell}(x),
$$

where

$$
\Theta_{m, \mu}(j, \ell)=\sum_{i=0}^{m} \eta_{i, m}(j) \Pi_{\mu}(j+i, \ell),
$$

and $\Pi_{\mu}(i, \ell), \eta_{i, m}(j)$, are defined in (10), (15), respectively.

Proof. The proofs of the this theorem can be immediately obtained on similar lines to that of Theorem 1 and Lemma 2.

Let us denote that

$$
\begin{gathered}
f_{k}=\left(f(x), \phi_{k}(x)\right)_{w_{t}, N}, \quad \mathbf{f}=\left(f_{0}, f_{1}, \ldots, f_{N-m}\right)^{T}, \\
u_{N}(x)=\sum_{\ell=0}^{N-m} a_{\ell} \phi_{\ell}(x), \quad \mathbf{a}=\left(a_{0}, a_{1}, \ldots, a_{N-m}\right)^{T}, \\
A=\left(a_{k j}\right)_{0 \leq k, j \leq N-m}, \quad B^{\sigma}=\left(b_{k j}^{\sigma}\right)_{0<k, j<N-m ; \sigma=1,2, \ldots, r-1}, \\
C=\left(c_{k j}\right)_{0 \leq k, j \leq N-m} .
\end{gathered}
$$

Then, the variational formulation (14) can be written as

$$
\begin{gathered}
\sum_{j=0}^{N} a_{j}\left[\left(D^{v} \phi_{j}(x), \phi_{k}(x)\right)_{w_{t}}+\sum_{\sigma=1}^{r-1} \gamma_{\sigma}\left(D^{\beta_{\sigma}} \phi_{j}(x), \phi_{k}(x)\right)_{w_{t}}\right. \\
\left.\quad+\gamma_{r}\left(\phi_{j}(x), \phi_{k}(x)\right)_{w_{t}}\right] \\
=\left(f, \phi_{k}(x)\right)_{w_{t}, N}, \quad k=0,1, \ldots, N-m .
\end{gathered}
$$

In view of (25), the Galerkin formulation (26) is equivalent to the following linear system:

$$
\left(A+\sum_{\sigma=1}^{r-1} \gamma_{\sigma} B^{\sigma}+\gamma_{r} C\right) \mathbf{a}=\mathbf{f},
$$

where the nonzero elements of the matrices $A, B^{\sigma}$ for $\sigma=$ $0,1, \ldots, r-1$ and $C$ are given explicitly in the following theorem.

Theorem 5. If one takes $\phi_{k}(x)$ as defined in (21), and if one denotes $a_{k j}=\left(D^{v} \phi_{j}(x), \phi_{k}(x)\right)_{w_{t}}, b_{k j}^{\sigma}=\left(D^{\beta_{\sigma}} \phi_{j}(x), \phi_{k}(x)\right)_{w_{t}}$, and $c_{k j}=\left(\phi_{j}(x), \phi_{k}(x)\right)_{w_{t}}$, then

$$
V_{N}=\operatorname{span}\left\{\phi_{0}(x), \phi_{1}(x), \ldots, \phi_{N-m}(x)\right\},
$$

and the elements $a_{k j}, b_{k j}^{\sigma}, c_{k j}$ for $0 \leq k, j \leq N-m$ are given by

$$
\begin{aligned}
a_{k j}= & \Theta_{m, v}(j, k) h_{k}+\sum_{l=l}^{m} \eta_{l, m}(k) \Theta_{m, v}(j, k+l) \hbar_{k+l}, \\
b_{k j}^{\sigma}= & \Theta_{m, \beta_{\sigma}}(j, k) h_{k}+\sum_{l=l}^{m} \eta_{l, m}(k) \Theta_{m, \beta_{\sigma}}(j, k+l) \hbar_{k+l}, \\
c_{k+p, k}= & c_{k, k+p} \\
= & \sum_{i=0}^{m-p}\left(\left(16(1+k)^{2}(3+2 k)^{2}(1+2 i+2 k)\right)\right. \\
& \times\left((1+i+2 k)_{3}(1+i+2 k+p)_{3}\right. \\
& \times(4-i) ! i ! \Gamma(3-i-p) \\
& \quad
\end{aligned}
$$

Proof. The basis functions $\phi_{k}(x)$ are chosen such that $\phi_{k}(x) \epsilon$ $V_{N}$ for $k=0,1, \ldots, N-m$, and the dimension of $V_{N}$ is equal to $N-m+1$. Hence,

$$
V_{N}=\operatorname{span}\left\{\phi_{0}(x), \phi_{1}(x), \ldots, \phi_{N-m}(x)\right\} .
$$

To obtain the elements $a_{k j}$ for $0 \leq k, j \leq N-m$, we set $\mu=\nu$ in Theorem 4 to get, for $j \geq\lceil\nu\rceil$,

$$
D^{\nu} \phi_{j}(x)=\sum_{s=0}^{\infty} \Theta_{m, v}(j, s) L_{t, s}(x),
$$

where $\Theta_{m, v}(j, s)$ is defined by relation (24). Due to (21) and (31), $a_{k j}$ takes the form

$$
\begin{aligned}
a_{k j}= & \left(\sum_{s=0}^{\infty} \Theta_{m, v}(j, s) L_{t, s}(x), L_{t, k}(x)\right)_{w_{t}} \\
& +\sum_{\ell=1}^{m} \eta_{\ell, m}(k)\left(\sum_{s=0}^{\infty} \Theta_{m, v}(j, s) L_{t, s}(x), L_{t, k+\ell}(x)\right)_{w_{t}} .
\end{aligned}
$$


Making use of the orthogonality relation (6), we obtain

$$
a_{k j}=\Theta_{m, \nu}(j, k) \hbar_{k}+\sum_{\ell=1}^{m} \eta_{\ell, m}(k) \Theta_{m, \nu}(j, k+\ell) \hbar_{k+\ell}
$$

this proves the first part of Theorem 5 . To prove the second part, we make use of relations (21) and (31), to obtain

$$
\begin{aligned}
b_{k j}^{\sigma}=( & \left.\sum_{s=0}^{\infty} \Theta_{m, \beta_{\sigma}}(j, s) L_{t, s}(x), L_{t, k}(x)\right)_{w_{t}} \\
& +\sum_{\ell=1}^{m} \eta_{\ell, m}(k)\left(\sum_{s=0}^{\infty} \Theta_{m, \beta_{\sigma}}(j, s) L_{t, s}(x), L_{t, k+\ell}(x)\right)_{w_{t}},
\end{aligned}
$$

then, it can be easily shown that

$$
b_{k j}^{\sigma}=\Theta_{m, \beta_{\sigma}}(j, k) \hbar_{k}+\sum_{\ell=1}^{m} \eta_{\ell, m}(k) \Theta_{m, \beta_{\sigma}}(j, k+\ell) \hbar_{k+\ell},
$$

which proves the second part of Theorem 5 . It can be shown, by using (21) and with the aid of (6), and after performing some manipulations, that the nonzero elements of $c_{k j}$ are given as in the following formula

$$
\begin{aligned}
c_{k+p, k} & =c_{k, k+p} \\
& =\sum_{i=0}^{m-p} \eta_{i+p, m}(k) \eta_{i, m}(k+p) \hbar_{k+i+p}, \quad 0 \leq p \leq m,
\end{aligned}
$$

and this proves the last part of the theorem and completes its proof.

Now, we will transform FDEs with nonhomogeneous initial conditions to other ones with homogeneous initial conditions. Consider the multi-term fractional differential equation (11) subject to the nonhomogeneous initial conditions

$$
u^{(j)}(0)=b_{j}, \quad j=0,1, \ldots, m-1 .
$$

Let us present the following transformation:

$$
V(x)=u(x)+\sum_{i=0}^{m-1} E_{i} x^{i}
$$

where

$$
E_{i}=\frac{-b_{i}}{i !}, \quad i=0,1, \ldots, m-1 .
$$

The transformation (38) turns the nonhomogeneous initial conditions (37) into the conditions

$$
V^{(j)}(0)=0, \quad j=0,1, \ldots, m-1 .
$$

Hence, it suffices to solve the following modified multi-term fractional differential equation:

$$
D^{\nu} V(x)+\sum_{\sigma=1}^{r-1} \gamma_{\sigma} D^{\beta_{\sigma}} V(x)+\gamma_{r} V(x)=f^{*}(x), \quad x \in I,
$$

subject to the homogeneous initial conditions (40), and

$$
f^{*}(x)=f(x)+\gamma_{r} \sum_{i=0}^{m-1} E_{i} x^{i}+\sum_{\sigma=1}^{r-1} \gamma_{\sigma} D^{\beta_{\sigma}}\left(\sum_{i=0}^{m-1} E_{i} x^{i}\right) .
$$

If we employ the shifted Legendre-Galerkin approximation to the modified problem (41), based on the basis function $\phi_{k}(x)$ which given in (21), we obtain the following system of linear algebraic equations:

$$
\left(A+\sum_{\sigma=1}^{r-1} \gamma_{\sigma} B^{\sigma}+\gamma_{r} C\right) \mathbf{a}=\mathbf{F}^{*}
$$

where $\mathbf{F}^{*}=\left(f_{0}^{*}, f_{1}^{*}, \ldots, f_{N-m}^{*}\right)^{T} ; f_{k}^{*}=\left(f^{*}, \phi_{k}(x)\right)_{w_{t}, N}$ and the elements of $A, B^{\sigma}$ for $\sigma=0,1, \ldots, r-1$ and $C$ are given in Theorem 5.

\section{Shifted Legendre Pseudospectral Approximation in Modal Basis}

The main advantage of pseudo-spectral approximation in solving differential equations $[30,31]$ lies in its high accuracy for a given number of unknowns. In the proposed shifted Legendre-Gauss-Lobatto collocation method in modal basis, there are two successive steps for obtaining the approximate solution of nonlinear fractional initial value problem. First, an appropriate finite set of shifted Legendre basis functions must be chosen for the representation of the truncated solution, and then the nonlinear FDE may be collocated by the well known shifted Legendre-Gauss-Lobatto quadrature nodes. Consequently, The nonlinear FDE is reduced to a system of algebraic equations. In the second step, we implement any standard numerical solver for solving such system of nonlinear algebraic equations.

In this section, we employ the shifted Legendre pseudospectral approximation in modal basis for the numerical solution the nonlinear fractional initial value problem:

$$
D^{v} u(x)=F\left(x, u(x), D^{\beta_{1}} u(x), \ldots, D^{\beta_{r}} u(x)\right), \quad x \in I,
$$

subject to the initial conditions (12), where $m-1<v \leq m$, $0<\beta_{1}<\beta_{2}<\cdots<\beta_{r}<\nu$. It is to be noted here that $F$ can be nonlinear in general.

If we denote by $x_{N, j}\left(x_{t, N, j}\right), 0 \leqslant j \leqslant N$, and $\omega_{N, j}\left(\omega_{t, N, j}\right)$, $0 \leq j \leq N$, the zeros and the weights of the standard (resp., shifted) Legendre-Gauss-Lobatto quadratures on $[-1,1]$ (resp., $[0,1])$, then we may deduce that

$$
\begin{aligned}
x_{t, N, j} & =\frac{1}{2}\left(x_{N, j}+1\right), \\
\omega_{t, N, j} & =\frac{1}{2} \omega_{N, j}, \\
0 & \leq j \leq N,
\end{aligned}
$$


and if $\grave{S}_{N}[0,1]$ denotes the set of all polynomials of degree $\leqslant N$, then for any $\psi \in \grave{S}_{2 N-1}[0,1]$, we get

$$
\begin{aligned}
& \int_{0}^{1} w_{t}(x) \psi(x) d x=\frac{1}{2} \int_{-1}^{1} w(x) \psi\left(\frac{1}{2}(x+1)\right) d x \\
& =\frac{1}{2} \sum_{j=0}^{N}{\omega_{N, j}} \psi\left(\frac{1}{2}\left(x_{N, j}+1\right)\right) \\
& =\sum_{j=0}^{N} \omega_{t, N, j} \psi\left(x_{t, N, j}\right),
\end{aligned}
$$

where $x_{N, j}$ are the zeros of $\left(1-x^{2}\right) \partial_{x} L_{N}$, and

$$
\varpi_{N, j}=\frac{2}{N(N+1)} \frac{1}{\left(L_{N}\left(x_{N, j}\right)\right)^{2}}, \quad 0 \leq j \leq N
$$

In fact, the discrete inner product and norm as are defined by

$$
\begin{gathered}
(u, v)_{w_{t}, N}=\sum_{k=0}^{N} u\left(x_{t, N, k}\right) v\left(x_{t, N, k}\right) \omega_{t, N, k}, \\
\|u\|_{w_{t}, N}=\sqrt{(u, u)_{w_{t}, N}} .
\end{gathered}
$$

Recalling

$$
\begin{gathered}
S_{N}=\operatorname{span}\left\{L_{t, 0}(x), L_{t, 1}(x), \ldots, L_{t, N}(x)\right\}, \\
V_{N}=\left\{v \in S_{N}: v^{(j)}(0)=0, j=0,1, \ldots, m-1\right\},
\end{gathered}
$$

then the shifted Legendre-Gauss-Lobatto collocation method for solving (44)-(12) is to seek $u_{N}(x) \in V_{N}$, such that

$$
\begin{array}{r}
D^{v} u\left(x_{t, N-m, k}\right) \\
=F\left(x_{t, N-m, k}, u\left(x_{t, N-m, k}\right),\right. \\
\left.D^{\beta_{1}} u\left(x_{t, N-m, k}\right), \ldots, D^{\beta_{r}} u\left(x_{t, N-m, k}\right)\right), \\
k=0,1, \ldots, N-m,
\end{array}
$$

where $x_{t, N-m, k}(k=0,1, \ldots, N-m)$ are the nodes of the shifted Legendre-Gauss-Lobatto quadratures on the interval $[0,1]$.

We now derive the algorithm for solving (44)-(12). To do this, let

$$
u_{N}(x)=\sum_{j=0}^{N-m} a_{j} \phi_{j}(x), \quad \mathbf{a}=\left(a_{0}, a_{1}, \ldots, a_{N-m}\right)^{T}
$$

where $\phi_{j}(x)$ are the shifted Legendre basis of functions defined in (16). The members of the basis may satisfy automatically the given initial conditions (12), imposed on the nonlinear FDEs. Then, by virtue of (51), we deduce that

$$
\begin{aligned}
& \sum_{j=0}^{N-m} a_{j} D^{v} \phi_{j}(x) \\
&=F\left(x, \sum_{j=0}^{N-m} a_{j} \phi_{j}(x),\right. \\
&\left.\quad \sum_{j=0}^{N-m} a_{j} D^{\beta_{1}} \phi_{j}(x), \ldots, \sum_{j=0}^{N-m} a_{j} D^{\beta_{r}} \phi_{j}(x)\right) .
\end{aligned}
$$

Making use of (16) and Theorem 4 (relation (23)) for approximating $D^{v} \phi_{j}(x), D^{\beta_{1}} \phi_{j}(x), \ldots, D^{\beta_{r}} \phi_{j}(x)$ in terms of the shifted Legendre polynomials. By substituting these approximations in (52), it yields

$$
\begin{aligned}
\sum_{j=0}^{N-m} a_{j}\left(\sum_{\ell=0}^{N} \Theta_{m, \mu}(j, \ell) L_{t, \ell}(x)\right) & \\
= & F\left(x, \sum_{j=0}^{N-m} a_{j}\left(\sum_{\ell=0}^{m} \eta_{\ell, m}(j) L_{t, j+\ell}(x)\right),\right. \\
& \sum_{j=0}^{N-m} a_{j}\left(\sum_{\ell=0}^{N} \Theta_{m, \beta_{1}}(j, \ell) L_{t, \ell}(x)\right), \ldots, \\
& \left.\sum_{j=0}^{N-m} a_{j}\left(\sum_{\ell=0}^{N} \Theta_{m, \beta_{r}}(j, \ell) L_{t, \ell}(x)\right)\right) .
\end{aligned}
$$

To find the solution $u_{N}(x)$, we collocate (53) at the GaussLobatto collocation points $x_{t, N-m, k}, k=0,1, \ldots, N-m$, yields

$$
\begin{gathered}
\sum_{j=0}^{N-m}\left(\sum_{\ell=0}^{N} \Theta_{m, \mu}(j, \ell) L_{t, \ell}\left(x_{t, N-m, k}\right)\right) a_{j} \\
=F\left(x_{t, N-m, k}, \sum_{j=0}^{N-m}\left(\sum_{\ell=0}^{m} \eta_{\ell, m}(j) L_{t, j+\ell}\left(x_{t, N-m, k}\right)\right) a_{j},\right. \\
\sum_{j=0}^{N-m}\left(\sum_{\ell=0}^{N} \Theta_{m, \beta_{1}}(j, \ell) L_{t, \ell}\left(x_{t, N-m, k}\right)\right) a_{j}, \ldots, \\
\left.\sum_{j=0}^{N-m}\left(\sum_{\ell=0}^{N} \Theta_{m, \beta_{r}}(j, \ell) L_{t, \ell}\left(x_{t, N-m, k}\right)\right) a_{j}\right) .
\end{gathered}
$$

Equation (54) constitutes a system of $N-m$ nonlinear algebraic equations in the unknown expansion coefficients $a_{j} ; j=0,1, \ldots, N-m$ which may be solved by using Newton's iteration method.

\section{Numerical Examples}

In this section, we implement several numerical examples to demonstrate the accuracy and applicability of the proposed 
TABLE 1: Maximum absolute error with various choices of $N$, for Example 1 .

\begin{tabular}{lcccccc}
\hline$N-6$ & $v$ & SLG method & $v$ & SLG method & $v$ & SLG method \\
\hline 8 & & $1.00 \times 10^{-3}$ & & $2.30 \times 10^{-3}$ & & $2.88 \times 10^{-3}$ \\
16 & 5.1 & $1.19 \times 10^{-7}$ & 5.5 & $1.11 \times 10^{-6}$ & 5.9 & $1.47 \times 10^{-6}$ \\
24 & & $7.15 \times 10^{-10}$ & & $1.08 \times 10^{-8}$ & & $1.86 \times 10^{-8}$ \\
\hline
\end{tabular}

spectral algorithms. Comparison of the results obtained by our methods with shifted Jacobi pseudo-spectral approximation [12] reveals that the present algorithms are very convenient and produces high accurate solutions to multiterm FDEs.

Example 1. Consider the linear FDE equation with homogeneous initial conditions

$$
\begin{aligned}
D^{v} u(x)+ & 5 D^{11 / 3} u(x)+3 D^{11 / 5} u(x) \\
- & 4 D^{11 / 7} u(x)-6 D^{11 / 13} u(x)=f(x), \\
& <<\leq 6, u^{(j)}(0)=0, j=0,1, \ldots, 5,
\end{aligned}
$$

where

$$
\begin{aligned}
f(x)= & \frac{31135104000 x^{28 / 3}}{\Gamma[31 / 3]}+\frac{18681062400 x^{54 / 5}}{\Gamma[59 / 5]} \\
& -\frac{24908083200 x^{80 / 7}}{\Gamma[87 / 7]}-\frac{37362124800 x^{158 / 13}}{\Gamma[171 / 13]} \\
& +\frac{6227020800 x^{13-v}}{\Gamma[14-\nu]} .
\end{aligned}
$$

The exact solution is given by $u(x)=x^{13}$.

Table 1 lists the maximum absolute error, using the shifted Legendre-Galerkin (SLG) method with various choices of $v$ and $N$.

Example 2. Consider the linear FDE equation

$$
\begin{array}{r}
D^{2} u(x)+D^{3 / 2} u(x)+D^{1 / 2} u(x)=g(x), \\
u(0)=1, u^{\prime}(0)=0, x \in[0,1],
\end{array}
$$

whose exact solution is given by $u(x)=\cos (\gamma x)$. The righthand side $g(x)$ can be obtained from the substitution of the exact solution in (57).

Table 2 lists the maximum absolute error, using the SLG method with various choices of $\gamma$ and $N$. Moreover, the approximate solution obtained by the SLG method at $\gamma=4 \pi$ and $N=16$ is shown in Figure 1 to make it easier to compare with the analytic solution.
TABLE 2: Maximum absolute error with various choices of $N$, for Example 2.

\begin{tabular}{lcccccc}
\hline$N-2$ & $v$ & SLG method & $v$ & SLG method & $v$ & SLG method \\
\hline 16 & & $2.87 \times 10^{-4}$ & & $3.21 \times 10^{-3}$ & & $3.99 \times 10^{-1}$ \\
20 & 1 & $2.47 \times 10^{-5}$ & $\pi$ & $2.43 \times 10^{-4}$ & $2 \pi$ & $9.96 \times 10^{-4}$ \\
24 & & $6.05 \times 10^{-6}$ & & $6.01 \times 10^{-5}$ & & $2.55 \times 10^{-4}$ \\
\hline
\end{tabular}

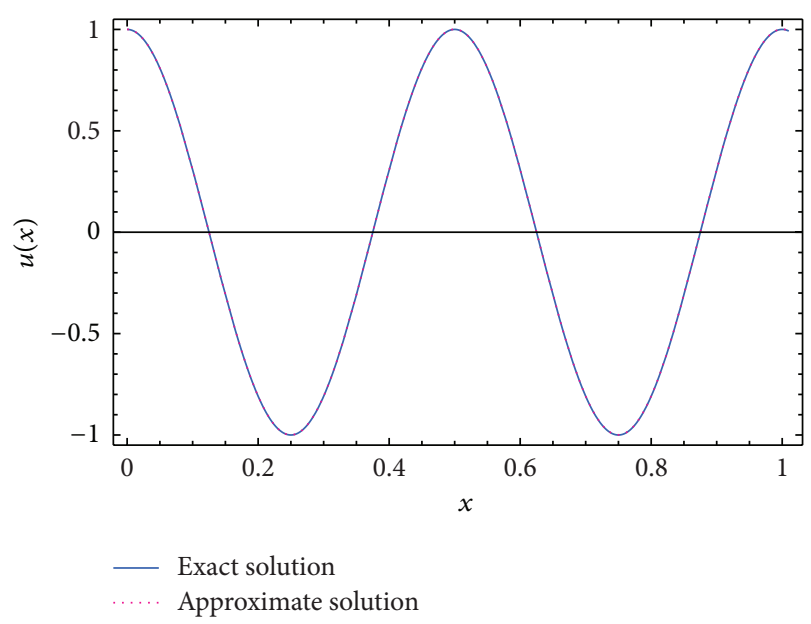

FIGURE 1: Comparison of $u_{N}(x)$, for $v=4 \pi$ and $N=16$, and $u(x)$, for Example 2.

Example 3. Consider the nonlinear fractional initial value problem [12]

$$
\begin{array}{r}
D^{\zeta} u(x)+D^{\eta} u(x) \cdot D^{\theta} u(x)+u^{2}(x) \\
=x^{6}+\frac{6 x^{3-\zeta}}{\Gamma(4-\zeta)}+\frac{36 x^{6-\eta-\theta}}{\Gamma(4-\eta) \Gamma(4-\theta)}, \\
\zeta \in(2,3), \eta \in(1,2), \theta \in(0,1), \\
u(0)=u^{\prime}(0)=u^{\prime \prime}(0)=0 .
\end{array}
$$

The exact solution is $u(x)=x^{3}$.

In Table 3, we introduce maximum absolute error, using SL-G-LC method for $\zeta=2.5, \eta=1.5, \theta=0.9$ with various choices of $N$.

This problem was solved in [12] using shifted JacobiGauss collocation (SJ-GC) method based on Jacobi operational matrix, the results provided by Doha et al. [12] have been presented in the third, fourth, and fifth columns of Table 3 for Jacobi parameters $\alpha=\beta=0, \alpha=\beta=1 / 2$, and $\alpha=\beta=1$, respectively. Numerical results of this FDE demonstrate that the SL-G-LC method is more accurate than the SJ-GC method, see Table 5.7 in [12]. 
TABLe 3: Maximum absolute error for $\zeta=2.5, \eta=1.5$, and $\theta=0.9$ and different choices of $N$, for Example 3.

\begin{tabular}{lcccc}
\hline$N$ & SL-G-LC & SJ-GC $(\alpha=\beta=0)$ & SL-GLC $(\alpha=\beta=1 / 2)$ & SL-GLC $(\alpha=\beta=1)$ \\
\hline 4 & $4.06 \times 10^{-4}$ & $3.91 \times 10^{-3}$ & $2.73 \times 10^{-3}$ & $1.84 \times 10^{-3}$ \\
8 & $1.61 \times 10^{-4}$ & $1.42 \times 10^{-3}$ & $8.66 \times 10^{-4}$ & $5.40 \times 10^{-4}$ \\
16 & $7.06 \times 10^{-5}$ & $1.24 \times 10^{-4}$ & $1.17 \times 10^{-4}$ & $1.03 \times 10^{-4}$ \\
24 & $2.33 \times 10^{-5}$ & $3.37 \times 10^{-5}$ & $3.50 \times 10^{-5}$ & $3.39 \times 10^{-5}$ \\
\hline
\end{tabular}

TABLE 4: Maximum absolute error with various choices of $N, m, \zeta$, and $p=4$, for Example 4 .

\begin{tabular}{lccccc}
\hline$N-m$ & $m$ & $\zeta$ & SL-G-LC & $\zeta$ & SL-G-LC \\
\hline 4 & & & $9.29 \times 10^{-3}$ & & $4.80 \times 10^{-3}$ \\
8 & \multirow{2}{*}{3} & 2.5 & $3.29 \times 10^{-3}$ & & $2.44 \times 10^{-3}$ \\
16 & & & $1.34 \times 10^{-3}$ & 2.9 & $2.65 \times 10^{-4}$ \\
24 & & & $4.89 \times 10^{-4}$ & & $2.63 \times 10^{-4}$ \\
\hline 4 & & & $1.16 \times 10^{-2}$ & & $1.98 \times 10^{-2}$ \\
8 & \multirow{2}{*}{5} & \multirow{2}{*}{4.2} & $3.69 \times 10^{-3}$ & & $4.31 \times 10^{-3}$ \\
16 & & & $1.47 \times 10^{-4}$ & 4.8 & $1.89 \times 10^{-3}$ \\
24 & & & $1.19 \times 10^{-4}$ & & $7.90 \times 10^{-4}$ \\
\hline
\end{tabular}

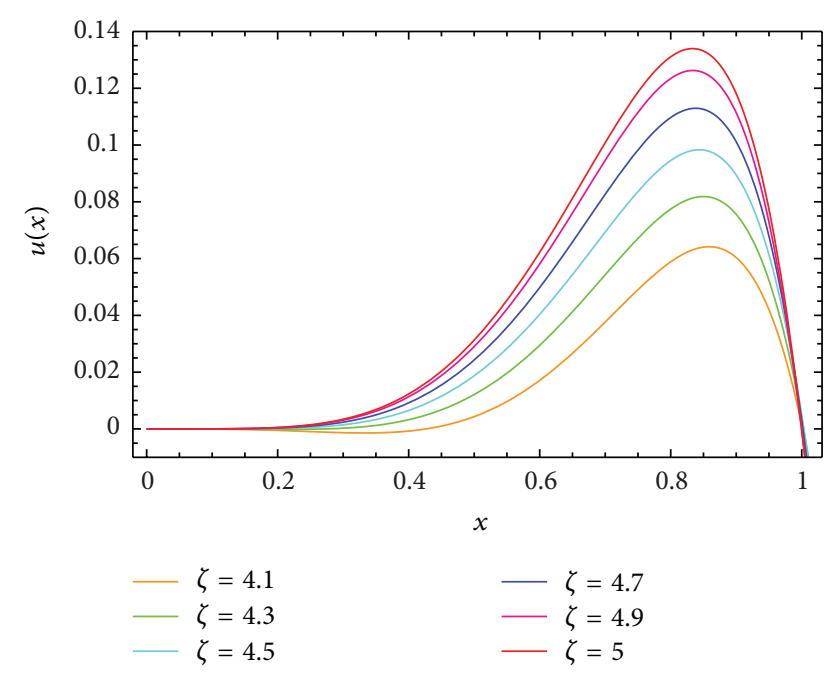

FIGURE 2: Approximated solution for $\zeta=4.1,4.3,4.5,4.7,4.9,5, p=$ 2 with 12 nodes, for Example 4.

Example 4. Consider the nonlinear fractional initial value problem

$$
\begin{array}{r}
D^{\zeta} u(x)+e^{u(x)}+u^{p}(x)=f(x), \quad \zeta \in(m-1, m), m \leq 5, \\
u^{j}(0)=0, j=0,1, \ldots, m-1,
\end{array}
$$

where

$$
\begin{aligned}
f(x)= & \frac{360 x^{5-\zeta}}{\Gamma(6-\zeta)}-\frac{1440 x^{6-\zeta}}{\Gamma(7-\zeta)}-\Gamma(1+\zeta) \\
& +\left(-x^{\zeta}+3 x^{5}-2 x^{6}\right)^{p}+e^{-x^{\zeta}+3 x^{5}-2 x^{6}}
\end{aligned}
$$

The exact solution of this problem is $u(x)=-x^{\zeta}+3 x^{5}-2 x^{6}$.

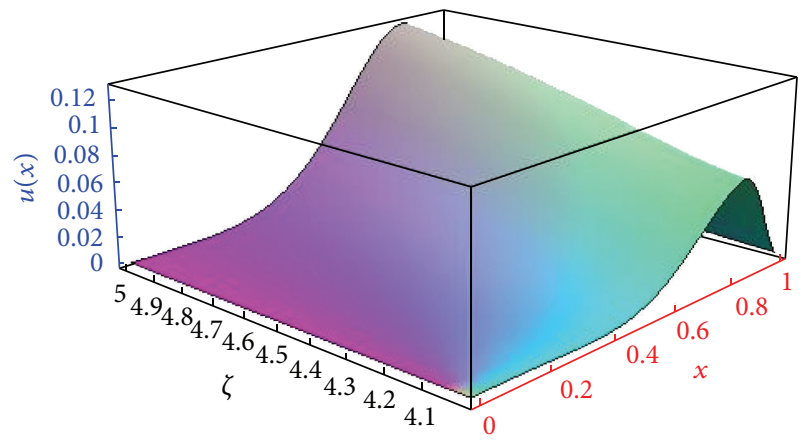

FIGURE 3: Approximated solution for $4<\zeta \leq 5, p=2$ with 12 nodes, for Example 4.

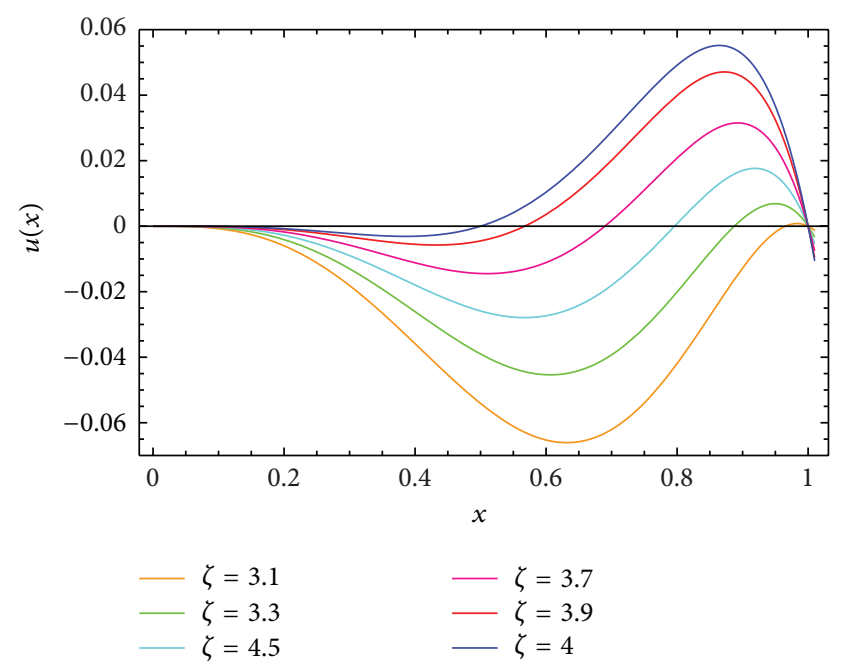

FIGURE 4: Approximated solution for $\zeta=3.1,3.3,3.5,3.7,3.9,5, p=$ 3 with 12 nodes, for Example 4.

In Table 4, we introduce the maximum absolute error, using the shifted Legendre collocation method based on Gauss-Lobatto points, with various choices of $\zeta, m$ and $N$ at $p=4$.

The approximated solutions are evaluated for $\zeta=$ $4.1,4.3,4.5,4.7,4.9,5$ with $m=5$ and $p=2$ and 12 nodes. The results of the numerical simulations are plotted in Figure 2. It is evident from Figure 2 that, as $\zeta$ approaches close to 5, the numerical solution by shifted Legendre-Gauss-Lobatto collocation method for such FDE approaches to the solution of integer order differential equation. In the case of $4<\zeta \leq 5$, $p=2$ with $m=5$, and 12 nodes, the results of the numerical simulations are shown in Figure 3. In Figure 4, we plotted 


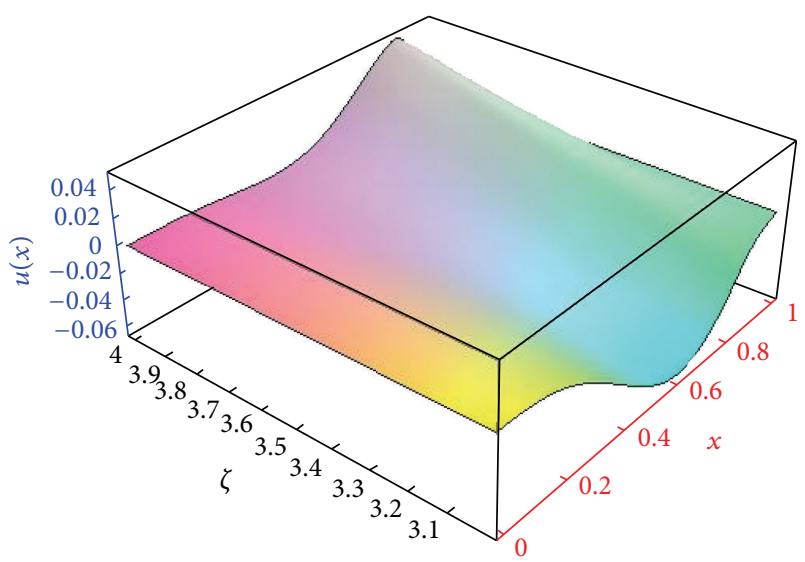

FIGURE 5: Approximated solution for $3<\zeta \leq 4, p=3$ with 12 nodes, for Example 4.

the approximated solutions for different choices of $\zeta, m=4$, $p=3$, and 12 nodes. Moreover, the approximate solutions obtained by the present method at $3<\zeta \leq 4, p=3$ with $m=4$, and 12 nodes are shown in Figure 5 to make it easier to show that; as $\zeta$ approaches to its integer value, the solution of FDE approaches to the solution of integer order differential equation.

\section{Conclusion}

We have extended the application of the shifted Legendre spectral Galerkin approximation for treating fractional initial value problems. In this approximation, the initial conditions are satisfied exactly for each member of shifted Legendre basis functions. In particular, any fractional-order Caputo derivative of such basis functions is expanded in terms of the shifted Legendre polynomials. In addition, we have proposed an accurate direct solvers for the general multi-term FDEs with nonhomogeneous initial conditions using the Legendre spectral Galerkin approximation.

In this paper, we proposed a Legendre-Gauss-Lobatto collocation algorithm in model basis for solving the nonlinear FDEs in which the numerical solution was approximated directly using the shifted Legendre basis functions. The results from numerical examples demonstrate the accuracy and stability of these spectral approximations for treating linear and nonlinear FDEs. In the forthcoming works, we hope that similar techniques can be applied to Chebyshev polynomials or other Jacobi polynomials.

\section{References}

[1] R. L. Magin, Fractional Calculus in Bioengineering, Begell House Publishers, 2006.

[2] S. Das, Functional Fractional Calculus for System Identification and Controls, Springer, New York, NY, USA, 2008.

[3] D. Baleanu, K. Diethelm, E. Scalas, and J. J. Trujillo, Fractional Calculus Models and Numerical Methods, vol. 3 of Series on Complexity, Nonlinearity and Chaos, World Scientific, 2012.
[4] I. Podlubny, Fractional Differential Equations, Academic Press, San Diego, Calif, USA, 1999.

[5] K. A. Gepreel, "The homotopy perturbation method applied to the nonlinear fractional Kolmogorov-Petrovskii-Piskunov equations," Applied Mathematics Letters, vol. 24, no. 8, pp. 1428 1434, 2011.

[6] S. Saha Ray and A. Patra, "An Explicit Finite Difference scheme for numerical solution of fractional neutron point kinetic equation," Annals of Nuclear Energy, vol. 41, pp. 61-66, 2012.

[7] D. Baleanu, A. K. Golmankhaneh, and A. K. Golmankhaneh, "Solving of the fractional nonlinear and linear Schrodinger equations by homotopy perturbation method," Romanian Journal of Physics, vol. 54, no. 10, 2009.

[8] A. Ansari, A. R. Sheikhani, and H. S. Najafi, "Solution to system of partial fractional differential equations using the fractional exponential operators," Mathematical Methods in the Applied Sciences, vol. 35, no. 1, pp. 119-123, 2012.

[9] E. H. Doha, A. H. Bhrawy, and S. S. Ezz-Eldien, "Efficient Chebyshev spectral methods for solving multi-term fractional orders differential equations," Applied Mathematical Modelling, vol. 35, no. 12, pp. 5662-5672, 2011.

[10] E. H. Doha, A. H. Bhrawy, and S. S. Ezz-Eldien, "A Chebyshev spectral method based on operational matrix for initial and boundary value problems of fractional order," Computers \& Mathematics with Applications, vol. 62, no. 5, pp. 2364-2373, 2011.

[11] S. Kazem, S. Abbasbandy, and S. Kumar, "Fractional-order Legendre functions for solving fractional-order differential equations," Applied Mathematical Modelling, vol. 37, pp. 5498-5510, 2013.

[12] E. H. Doha, A. H. Bhrawy, and S. S. Ezz-Eldien, "A new Jacobi operational matrix: an application for solving fractional differential equations," Applied Mathematical Modelling, vol. 36, no. 10, pp. 4931-4943, 2012.

[13] A. Ahmadian, M. Suleiman, S. Salahshour, and D. Baleanu, "A Jacobi operational matrix for solving fuzzy linear fractional differential equation," Advances in Difference Equations, vol. 2013, article 104, 2013.

[14] M. Maleki, I. Hashim, M. Tavassoli Kajani, and S. Abbasbandy, "An adaptive pseudospectral method for fractional order boundary value problems," Abstract and Applied Analysis, vol. 2012, Article ID 381708, 19 pages, 2012.

[15] A. Al-Rabtah, S. Momani, and M. A. Ramadan, "Solving linear and nonlinear fractional differential equations using spline functions," Abstract and Applied Analysis, vol. 2012, Article ID 426514, 9 pages, 2012.

[16] A. H. Bhrawy and M. Alshomrani, "A shifted Legendre spectral method for fractional-order multi-point boundary value problems," Advances in Difference Equations, vol. 2012, article 8, 2012.

[17] S. Yüzbaşı, "Numerical solution of the Bagley-Torvik equation by the Bessel collocation method," Mathematical Methods in the Applied Sciences, vol. 36, no. 3, pp. 300-312, 2013.

[18] S. Yüzbaşı, "Numerical solutions of fractional Riccati type differential equations by means of the Bernstein polynomials," Applied Mathematics and Computation, vol. 219, no. 11, pp. 6328-6343, 2013.

[19] A. H. Bhrawy, M. M. Alghamdi, and T. M. Taha, "A new modified generalized Laguerre operational matrix of fractional integration for solving fractional differential equations on the half line," Advances in Difference Equations, vol. 2012, article 179, 2012. 
[20] D. Baleanu, A. H. Bhrawy, and T. M. Taha, "Two efficient generalized Laguerre spectral algorithms for fractional initial value problems," Abstract and Applied Analysis, vol. 2013, Article ID 546502, 10 pages, 2013.

[21] B. Bandrowski, A. Karczewska, and P. Rozmej, "Numerical solutions to fractional perturbed Volterra equations," Abstract and Applied Analysis, vol. 2012, Article ID 529602, 19 pages, 2012.

[22] Y.-L. Jiang and X.-L. Ding, "Waveform relaxation methods for fractional differential equations with the Caputo derivatives," Journal of Computational and Applied Mathematics, vol. 238, pp. 51-67, 2013.

[23] A. Ansari, "Fractional exponential operators and time-fractional telegraph equation," Boundary Value Problems, vol. 2012, article 125, 2012.

[24] A. G. Butkovskii, S. S. Postnov, and E. A. Postnova, "Fractional integro-differential calculus and its control-theoretical applications. I. Mathematical fundamentals and the problem of interpretation," Automation and Remote Control, vol. 74, pp. 543-574, 2013.

[25] X. Zhang, C. Zhu, and Z. Wu, "Solvability for a coupled system of fractional differential equations with impulses at resonance," Boundary Value Problems, vol. 2013, article 80, 2013.

[26] M. Shaban, S. Kazemb, and J. A. Rad, "A modification of the homotopy analysis method based on Chebyshev operational matrices," Mathematical and Computer Modelling, vol. 57, pp. 1227-1239, 2013.

[27] E. H. Doha, A. H. Bhrawy, D. Baleanu, and S. S. Ezz-Eldien, "On shifted Jacobi spectral approximations for solving fractional differential equations," Applied Mathematics and Computation, vol. 219, no. 15, pp. 8042-8056, 2013.

[28] E. H. Doha, A. H. Bhrawy, and W. M. Abd-Elhameed, "Jacobi spectral Galerkin method for elliptic Neumann problems," Numerical Algorithms, vol. 50, no. 1, pp. 67-91, 2009.

[29] E. H. Doha and A. H. Bhrawy, "An efficient direct solver for multidimensional elliptic Robin boundary value problems using a Legendre spectral-Galerkin method," Computers \& Mathematics with Applications, vol. 64, no. 4, pp. 558-571, 2012.

[30] C. Canuto, M. Y. Hussaini, A. Quarteroni, and T. A. Zang, Spectral Methods in Fluid Dynamics, Springer, New York, NY, USA, 1988.

[31] A. H. Bhrawy and A. S. Alofi, "A Jacobi-Gauss collocation method for solving nonlinear Lane-Emden type equations," Communications in Nonlinear Science and Numerical Simulation, vol. 17, no. 1, pp. 62-70, 2012. 


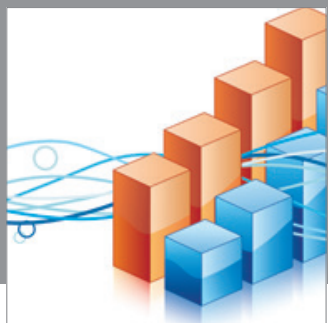

Advances in

Operations Research

mansans

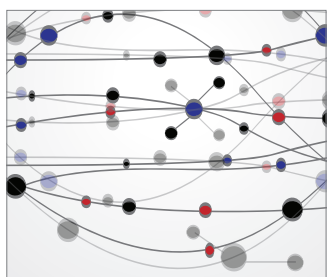

The Scientific World Journal
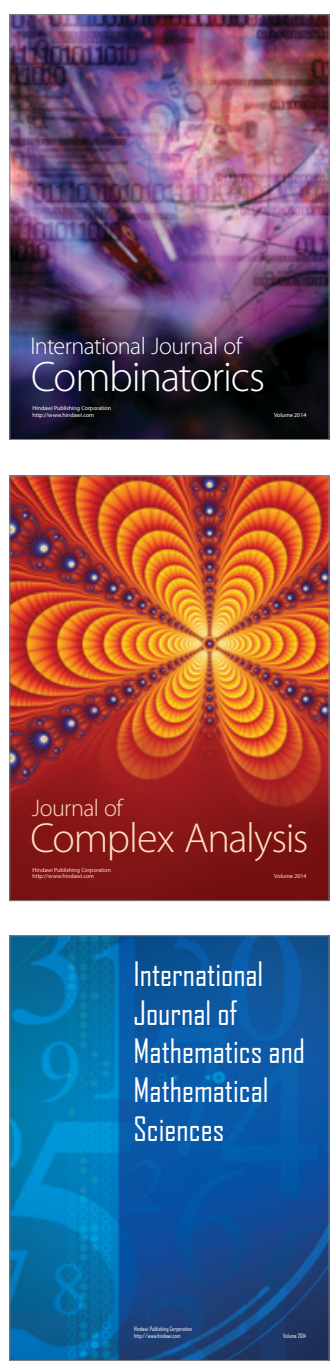
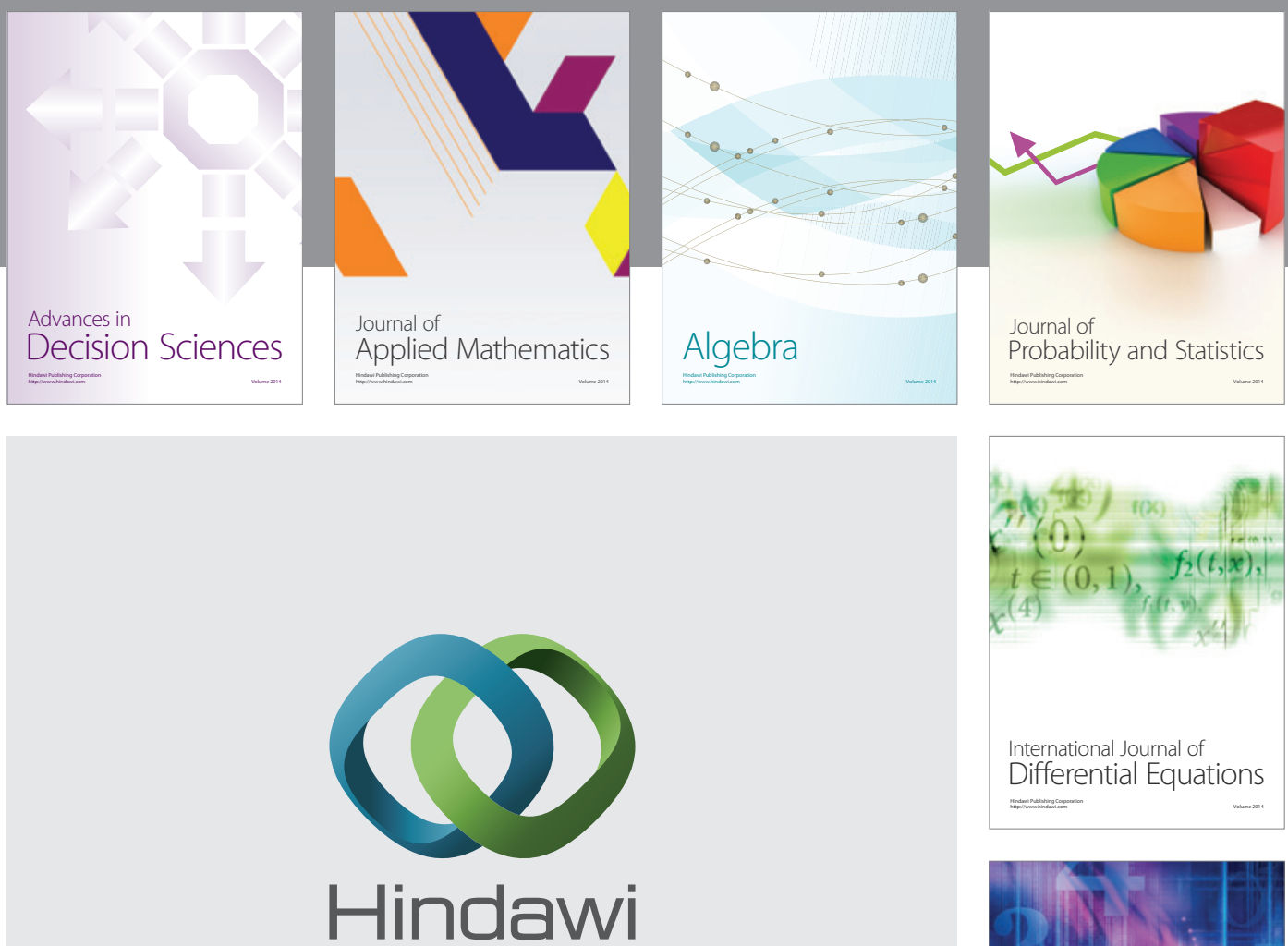

Submit your manuscripts at http://www.hindawi.com
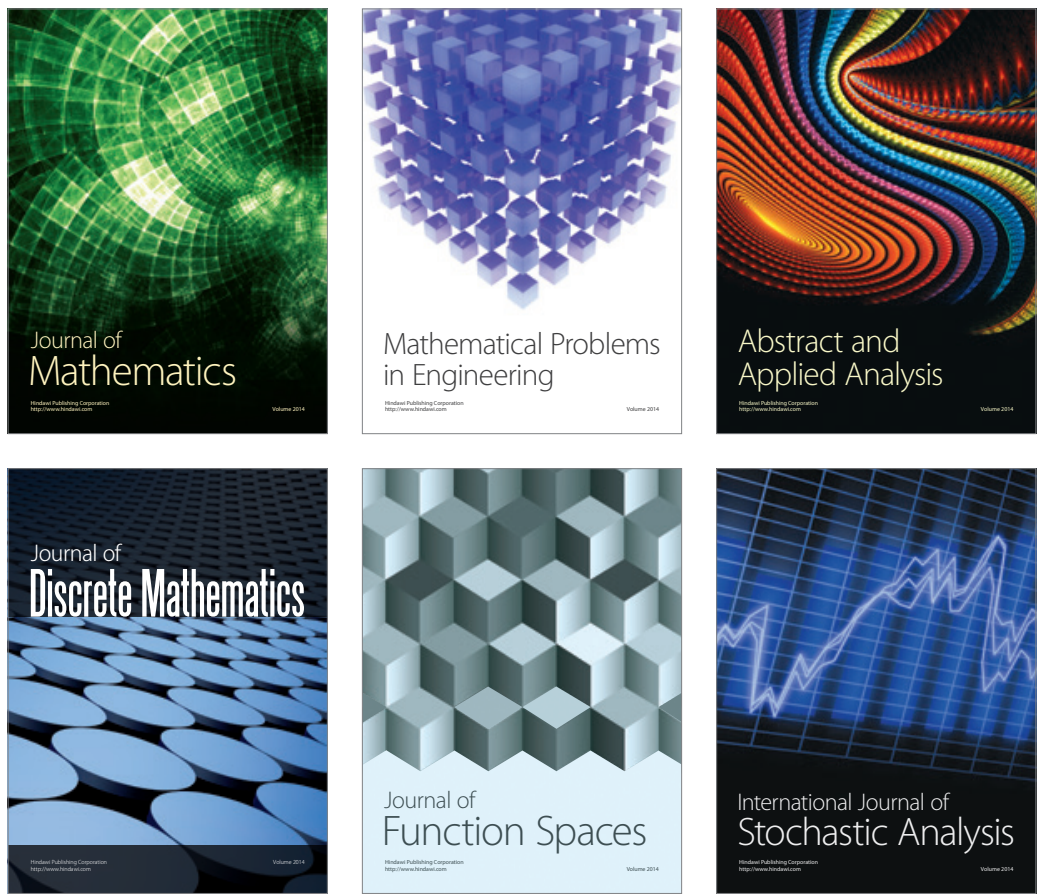

Journal of

Function Spaces

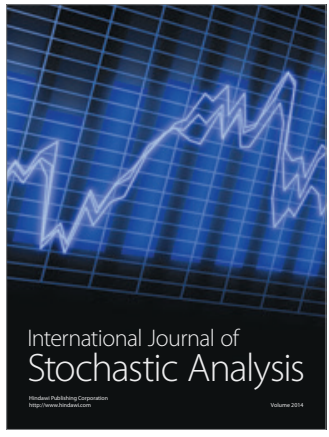

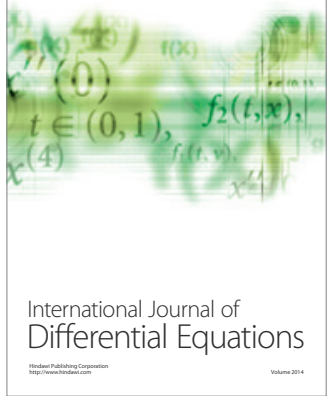
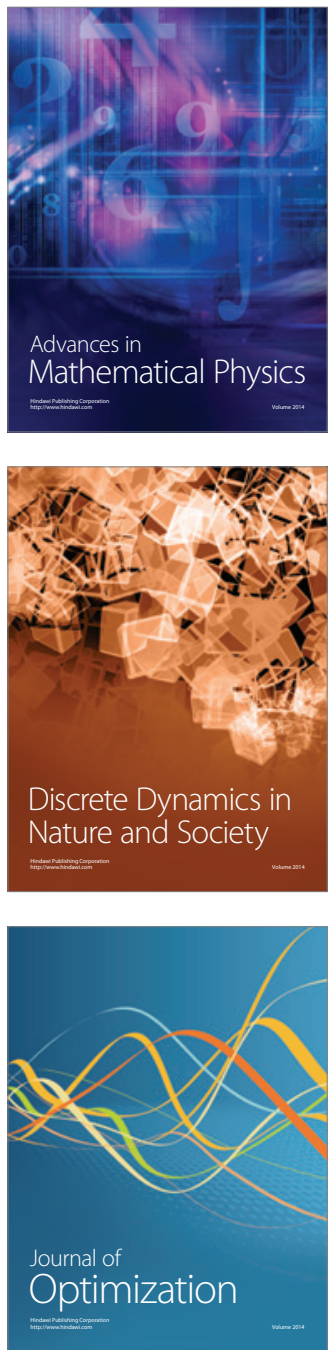